\title{
Banxia Xiexin Decoction Ameliorates t-BHP-Induced Apoptosis in Pancreatic Beta Cells by Activating the PI3K/AKT/FOXO1 Signaling Pathway
}

\author{
Li-juan Du, ${ }^{1}$ Bing Pang $\mathbb{D},{ }^{1}$ Yu-meng Tan $\mathbb{D}^{1},{ }^{1}$ Ya-nan Yang $\mathbb{D},{ }^{1}$ Mei-zhen Zhang $\mathbb{D},{ }^{1}$ \\ Qing Pang, ${ }^{1}$ Min Sun $\mathbb{D}^{2}$, and Qing Ni ${ }^{1}$ \\ ${ }^{1}$ Department of Endocrinology, Guang'anmen Hospital of China Academy of Chinese Medical Sciences, Beijing, China \\ ${ }^{2}$ School of Life Sciences, Anhui University, Hefei, China \\ Correspondence should be addressed to Min Sun; sm3216@126.com and Qing Ni; niqing669@163.com
}

Li-juan Du and Bing Pang contributed equally to this work.

Received 27 December 2019; Revised 20 March 2020; Accepted 31 March 2020; Published 21 April 2020

Academic Editor: Eric Hajduch

Copyright (C) $2020 \mathrm{Li}$-juan Du et al. This is an open access article distributed under the Creative Commons Attribution License, which permits unrestricted use, distribution, and reproduction in any medium, provided the original work is properly cited.

Background. Banxia Xiexin Decoction (BXXD) reportedly regulates glycolipid metabolism and inhibits pancreatic $\beta$-cell apoptosis. This study is aimed at investigating the protective effect of BXXD on tert-butyl hydroperoxide- (t-BHP-) induced apoptosis in MIN6 cells and the underlying mechanisms. Methods. MIN6 cells were preincubated with BXXD or liraglutide (Li) with or without PI3K inhibitor LY294002 (LY) for $12 \mathrm{~h}$, following which t-BHP was added to induce MIN6 cell apoptosis. The protective effects of BXXD on MIN6 cells were evaluated by detecting cell viability and proliferation and glucose-stimulated insulin secretion (GSIS). The antiapoptotic effects were evaluated by Hoechst 33342 staining and terminal deoxynucleotidyl transferase dUTP nick end labeling assay (TUNEL). Malondialdehyde and glutathione peroxidase content and superoxide dismutase activity were measured using commercial kits. The expression of PI3K/AKT/FOXO1 signaling pathway-related signal molecules, and that of apoptotic indicators Bax, P27, and Caspase-3, was quantified using western blotting. Results. Preincubation with BXXD significantly improved t-BHP-induced proliferation inhibition and apoptosis and enhanced GSIS. t-BHP induced the generation of reactive oxygen species and inhibited the activities of antioxidant enzymes, which could be neutralized by pretreatment with BXXD. BXXD promoted the phosphorylation of AKT and FOXO1 in t-BHP-induced MIN6 cells. Moreover, BXXD attenuated the expression of related apoptotic indicators Bax, P27, and Caspase-3. LY abolished these effects of BXXD. Conclusion. BXXD protected MIN6 cells against $\mathrm{t}$-BHP-induced apoptosis and improved insulin secretory function through modulation of the PI3K/AKT pathway and the downstream FOXO1, thus suggesting a novel therapeutic approach for type 2 diabetes mellitus (T2DM).

\section{Introduction}

The dramatic increase in the incidence of diabetes mellitus $(\mathrm{DM})$ is becoming a major public health issue [1]. China reported 415 million patients with diabetes in 2015, and it is estimated that this number will reach 640 million by the year 2040 [2]. Diabetic complications are severe and irreversible, dramatically affecting the quality of life of the patients. Moreover, the expenses of diabetic vascular complications account for $80 \%$ of the total direct medical expenses, resulting in a large economic burden for society [3-5]. Therefore, the development of strategies for early prevention and treatment is necessary.

Pancreatic $\beta$-cell apoptosis plays a vital role in the development of T2DM [6]. Enhanced pancreatic $\beta$-cell apoptosis leads to $\beta$-cell loss in T2DM, which is characterized by progressive deterioration and ultimate failure of $\beta$-cell function. Reversal of $\beta$-cell function is critical for islet function, such as in maintenance of healthy glucose metabolism $[7,8]$. Oxidative stress damage may be an important factor resulting in pancreatic $\beta$-cell apoptosis. The PI3K/AKT signaling pathway, as a classical signaling pathway, plays an important role 
in the growth, proliferation, differentiation, and metabolism of pancreatic $\beta$-cells. PI3K, stimulated by a series of upstream molecules, acts on the downstream target of AKT and regulates its phosphorylation, leading to alterations in the expression of a protein belonging to the Bcl-2 family and the activation of caspase, which plays an important role in regulating $\beta$-cell apoptosis [9-12].

Banxia Xiexin Decoction is a well-known traditional Chinese herbal formula containing seven herbs and is commonly used to treat gastrointestinal diseases, diabetes, and related diseases [13-16]. Our previous studies demonstrated that BXXD lowers blood glucose, inhibits pancreatic $\beta$-cell apoptosis, attenuates oxidative stress, and promotes glucagonlike peptide 1 secretion $[17,18]$. However, the mechanisms underlying the action of BXXD have not been elucidated. In this study, we aimed to explore the mechanisms by which BXXD modulates pancreatic $\beta$-cell apoptosis and insulin secretion through the PI3K/AKT/FOXO1 signaling pathway, thus providing novel scientific evidence for traditional Chinese medicine therapy.

\section{Materials and Methods}

2.1. Cell Culture. Mouse MIN6 pancreatic $\beta$-cells were obtained from the American Type Culture Collection (ATCC). MIN6 cells were grown in high-glucose Dulbecco's modified Eagle's medium (DMEM) (HyClone, USA) containing $25 \mathrm{mmol} / \mathrm{l}$ glucose supplemented with $15 \%$ fetal bovine serum (FBS) (Lonza, Switzerland), $100 \mathrm{U} / \mathrm{ml}$ penicillin, $100 \mu \mathrm{g} / \mathrm{ml}$ streptomycin, and $5 \mu \mathrm{l} / \mathrm{l} \beta$-mercaptoethanol and cultured in a humidified atmosphere containing 5\% $\mathrm{CO}_{2}$ at $37^{\circ} \mathrm{C}$.

2.2. MTT Assay. The cytotoxicity of MIN6 cells was determined by a 3-(4,5-dimethylthiazol-2-yl)-2,5-diphenyltetrazolium bromide (MTT) assay. MIN6 cells were seeded into 96-well plates at a density of $5 \times 10^{3}$ cells/well. For determining the cytotoxicity of BXXD, the cells were pretreated with different concentrations of $\operatorname{BXXD}(0.1,0.5,1,1.5,2.5,5$, and $10 \mathrm{mg} / \mathrm{ml}$ ) for $12 \mathrm{~h}$. Subsequently, $100 \mu \mathrm{M}$ t-BHP was added, followed by incubation for $2 \mathrm{~h}$. Then, the MTT solution $(0.5 \mathrm{mg} / \mathrm{ml})$ was added to each well, followed by incubation for $4 \mathrm{~h}$ at $37^{\circ} \mathrm{C}$. The supernatant was discarded, $150 \mu \mathrm{l}$ Dimethyl sulfoxide (DMSO) for each well, and the cells were shaken for $15 \mathrm{~min}$. Absorbance (OD value) at a wavelength of $490 \mathrm{~nm}$ was measured using a microplate reader. MIN6 cell viability (\%) was calculated based on the absorbance of the treatment sample group/absorbance of the control group $\times 100$.

2.3. Cell Apoptosis Assay. MIN6 cell apoptosis was detected using the chromatin-specific dye Hoechst 33342 (Sigma, Germany) staining and TUNEL (Shanghai Beyotime, China) assay. After pretreatment with BXXD $(0.5 \mathrm{mg} / \mathrm{ml}), \mathrm{Li}$ $(50 \mathrm{Nm}), \mathrm{LY}(100 \mu \mathrm{M}), \mathrm{Li}+\mathrm{LY}$, or BXXD+LY for $12 \mathrm{~h}$, $100 \mu \mathrm{M}$ t-BHP (Tianjin Alfa Aesar, China) was added, followed by incubation for $2 \mathrm{~h}$. The cells were then washed three times with phosphate-buffered saline (PBS) (HyClone, USA). For Hoechst staining, $5 \mu \mathrm{g} / \mathrm{ml}$ Hoechst was added, followed by incubation for $20 \mathrm{~min}$. The dye solution was then allowed to absorb, followed by washing three times with PBS. For the TUNEL staining, MIN6 cells were fixed with a $4 \%$ paraformaldehyde solution for $30 \mathrm{~min}$ at room temperature and then permeabilized with $0.3 \%$ Triton X-100 for $5 \mathrm{~min}$. Following a wash with PBS, samples were first incubated with terminal deoxynucleotide TUNEL reagent containing terminal deoxynucleotidyl transferase and fluorescein isothiocyanatedUTP. The cells were then counterstained with $1 \mu \mathrm{g} / \mathrm{ml} 4^{\prime}, 6-$ diamidino-2-phenylindole (DAPI) for $30 \mathrm{~min}$. Images were taken using a fluorescence microscope.

2.4. Glucose-Stimulated Insulin Secretion (GSIS). MIN6 cells were seeded in 24-well plates in triplicate, treated with BXXD, Li, or LY for $12 \mathrm{~h} .100 \mu \mathrm{M} \mathrm{t}-\mathrm{BHP}$ was added, followed by incubation for $2 \mathrm{~h}$. Cells were preincubated in $0.5 \mathrm{ml}$ Krebs-Ringer bicarbonate (KRB) buffer containing $2.8 \mathrm{mM}$ glucose for $1 \mathrm{~h}$. The KRB buffer was removed and replaced by $0.5 \mathrm{ml} \mathrm{KRB}$ buffer containing $2.8 \mathrm{mM}$ glucose for $1 \mathrm{~h}$ (this was considered the basal level). The islets were then incubated in KRB buffer containing $16.7 \mathrm{mM}$ glucose for $1 \mathrm{~h}$ (this was considered the stimulated level). Insulin contents were determined using a mouse insulin ELISA kit (Mercodia, Uppsala, Sweden). The insulin stimulatory index of islets was calculated as stimulated/basal insulin secretion.

2.5. Estimation of Oxidative Stress Parameters (MDA, SOD, and GSH-Px). For the measurement of malondialdehyde (MDA), cells were grown in flasks. After treatment with the indicated drugs for $12 \mathrm{~h}$, the cells were subsequently treated with $100 \mu \mathrm{M} \mathrm{t}$-BHP for $2 \mathrm{~h}$. Then, the cells were harvested and lysed for the detection of MDA content. For detecting the activity of superoxide dismutase (SOD) and glutathione peroxidase (GSH-Px), cells were seeded in 24-well plates and treated as mentioned above. The culture media were collected for SOD and GSH-Px assays. The MDA, SOD, and GSH-Px levels were measured using commercial kits (Nanjing Jiancheng, China) according to the manufacturer's instructions. The protein concentration of the cell lysates was determined using a bicinchoninic acid (BCA) assay kit. The MDA content was normalized by the cellular protein content.

2.6. Intracellular Reactive Oxygen Species (ROS) Detection. Intracellular ROS levels were measured using a dichlorodihydro-fluorescein diacetate (DCFH-DA) probe (Sigma, Germany). MIN6 cells $\left(5 \times 10^{3}\right)$ were seeded in quadruplicate into a 96-well plate and treated as mentioned above. Cells were incubated in DMEM (phenol red-free) containing $10 \mu \mathrm{M}$ DCFH-DA at $37^{\circ} \mathrm{C}$ for $30 \mathrm{~min}$ and then washed three times with PBS to remove the residual probe. The cells were then washed with DMEM without phenol red. The fluorescence intensities were detected using a fluorescence microplate reader (BioTek, USA) at Ex 485/Em $530 \mathrm{~nm}$.

2.7. Western Blotting. After treatment, MIN6 cells were harvested, washed with cold PBS 3 times, and incubated in RIPA lysis buffer on ice for $20 \mathrm{~min}$. The lysis conditions were as follows: $12000 \mathrm{rpm}$ at $4^{\circ} \mathrm{C}$. Protein content was quantified using a BCA assay kit (Thermo Scientific, 




(a)

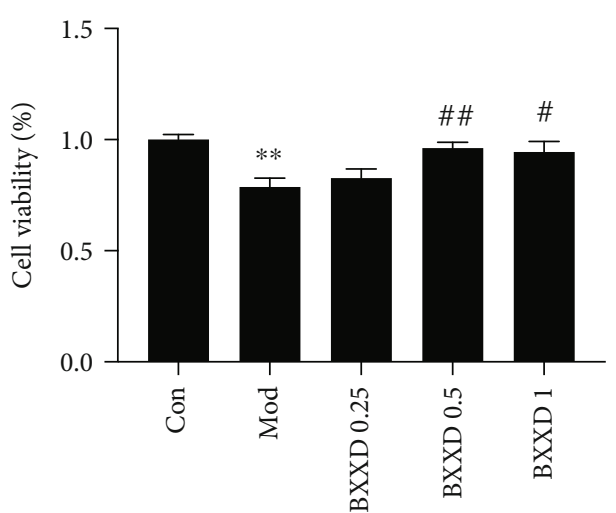

(b)

FIGURE 1: Determination of BXXD experiment conditions. (a) MIN6 cells were preconditioned with different concentrations of BXXD (0.1, $0.5,1,1.5,2.5,5$, and $10 \mathrm{mg} / \mathrm{ml})$ for $12 \mathrm{~h}$. (b) MIN6 cells were pretreated with BXXD $(0.25,0.5$, and $1 \mathrm{mg} / \mathrm{ml})$ for $12 \mathrm{~h}$ and then treated with $100 \mu \mathrm{M}$ t-BHP for $2 \mathrm{~h}$. Cell viability was detected by MTT assay. MIN6 cells were incubated with DMEM (Con), DMEM plus t-BHP (Mod), and DMEM, t-BHP plus different concentrations of BXXD (BXXD 0.25, BXXD 0.5, and BXXD 1). Values are expressed as mean \pm SEM. The experiment was repeated three times. ${ }^{* *} P<0.01$ versus Con; ${ }^{*} P<0.05$ versus Con; ${ }^{\# \# ~} P<0.01$ versus Mod, and ${ }^{\#} P<0.05$ versus Mod.

China). After the loading buffer was added and mixed, the sample was boiled for $10 \mathrm{~min}$, cooled, and stored in a $-20^{\circ} \mathrm{C}$ refrigerator. Aliquots were separated by $10 \%$ sodium dodecyl sulfate-polyacrylamide gel electrophoresis (SDS-PAGE) and then transferred to polyvinylidene fluoride membranes (Bio-Rad, USA). Membranes were blocked using SuperBlock T20 (TBS) buffer for $1 \mathrm{~h}$, then incubated with primary antibodies overnight at $4^{\circ} \mathrm{C}$, and washed three times with TBST. The dilution ratio used was 1:500, and the proteins were as follows: p27 (Cell Signaling Technology, USA), AKT (Cell Signaling Technology, USA), AKT (Phospho-Ser473) (Cell Signaling Technology, USA), FOXO1 (Cell Signaling Technology, USA), FOXO1 (phospho Ser256) (Cell Signaling Technology, USA), Bax (Proteintech, USA), and Caspase-3 (Proteintech, USA). Quantification of protein expression was normalized to that of $\beta$-actin (Shanghai Abmart, China) $(1: 5000)$. The membranes were then incubated with horseradish peroxidase-conjugated secondary antibodies for $2 \mathrm{~h}$ at room temperature. The FluorChem ${ }^{\mathrm{TM}} \mathrm{E}$ System (ProteinSimple, USA) was used to visualize bands of the antigen-antibody complexes, and the ImageJ software (NIH, USA) was employed for densitometric analysis to obtain band intensities.

2.8. Statistical Analysis. All data were representative of at least three separate experiments. The values were expressed as mean \pm SEM, and images were presented by GraphPad Prism 7.0 Software (GraphPad Software, USA). A one-way analysis of variance (ANOVA) with the Bonferroni test and Tukey test was used to compare between groups. SPSS version 20.0 (IBM Corp., Armonk, NY, USA) was used for the analyses. Values of $P<0.05$ was considered to be statistically significant.

\section{Results}

3.1. Cytotoxicity and Cell Proliferation in MIN6 Cell Exposed to BXXD. No evident cytotoxic effects of BXXD were observed at doses of $0.1-1.5 \mathrm{mg} / \mathrm{ml}$; BXXD reduced cell via- bility from 2.5 to $10 \mathrm{mg} / \mathrm{ml}$ (Figure $1(\mathrm{a})$ ). Therefore, we further studied whether BXXD could reverse t-BHP-induced cell proliferation inhibition. Cells were preincubated with different concentrations of BXXD $(0.25,0.5$, and $1 \mathrm{mg} / \mathrm{ml})$ and then stimulated with $100 \mu \mathrm{M}$ t-BHP for $2 \mathrm{~h}$. As shown (Figure 1(b)), t-BHP stimulation significantly reduced cell proliferation, and BXXD preincubation increased cell proliferation in an approximately dose-dependent manner. Therefore, the concentration of $0.5 \mathrm{mg} / \mathrm{ml}$ was used in subsequent experiments.

3.2. BXXD Protected MIN6 Cells by Reducing MIN6 Cell Apoptosis. Hoechst 33342 staining revealed that cells of the control group were intact and their nuclei were evenly stained. After t-BHP stimulation, the cells were irregularly condensed and agglomerated, showing dense staining and the nucleus splitting into fragments. Compared with the model group, the BXXD and liraglutide group showed alleviated nuclear condensation and reduced cell debris. Besides, when LY294002 was added, the protective effect of BXXD and liraglutide was not found to be ideal (Figures 2(a) and 2(b)). t-BHP induction markedly induced apoptosis in MIN6 cells; the percentage of apoptotic cells markedly increased in the model group compared to the control group $(9.84 \pm 1.00 \%$ versus $5.50 \pm 0.22 \%, P<0.05)$. Cotreatment of MIN6 cells with $100 \mu \mathrm{M}$ t-BHP and BXXD $(0.5 \mathrm{mg} / \mathrm{ml})$ for $12 \mathrm{~h}$ reduced the percentage of apoptotic cells to $7.35 \pm 0.08$ $\%(P<0.05)$. The positive cell content rate for the liraglutide group was $6.31 \pm 0.32 \%(P<0.05)$. A significant difference was found between the BXXD group and the liraglutide group $(P<0.05)$. In addition, when LY294002 was preadded, BXXD and liraglutide could not improve the apoptosis induced by t-BHP (Figure 2(c)).

3.3. BXXD Rectified High-Glucose-Stimulated Insulin Secretion. To investigate the effect of BXXD on $\beta$-cell function, insulin secretion in response to glucose stimulation was detected. GSIS was impaired in t-BHP-treated MIN6 cells. As shown in Figure 3,16.7 mM glucose failed to 

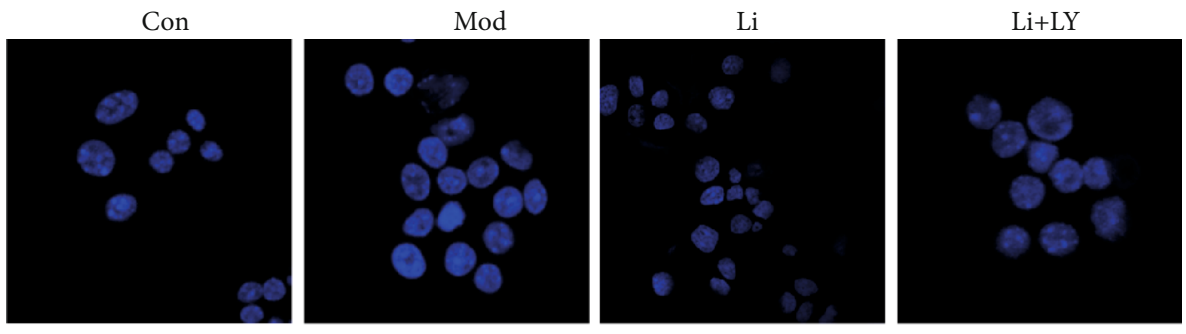

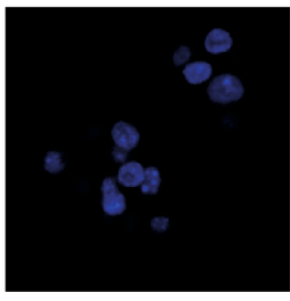

BXXD

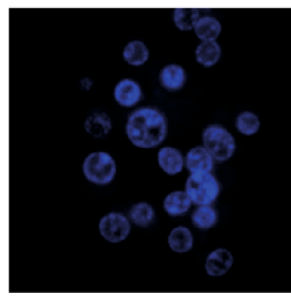

BXXD+LY

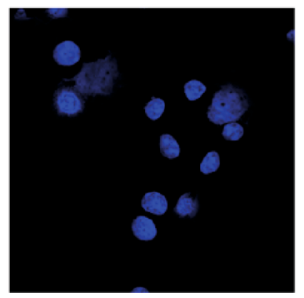

LY

(a)
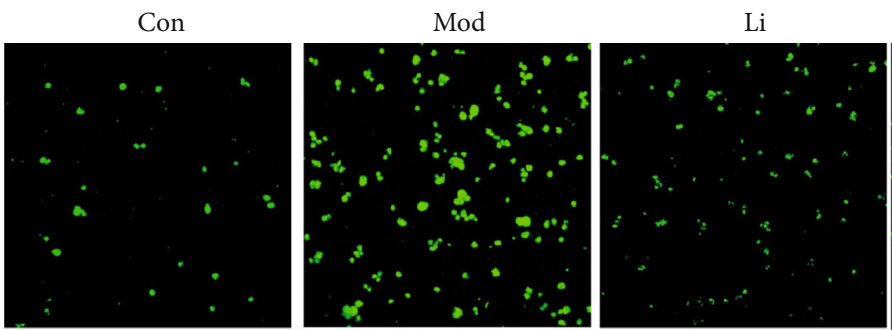

$\mathrm{Li}+\mathrm{LY}$

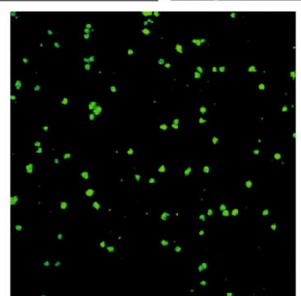

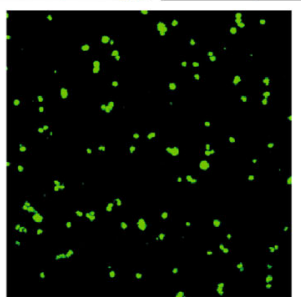

BXXD+LY

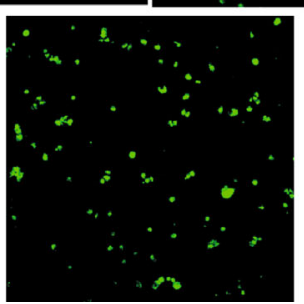

LY

(b)

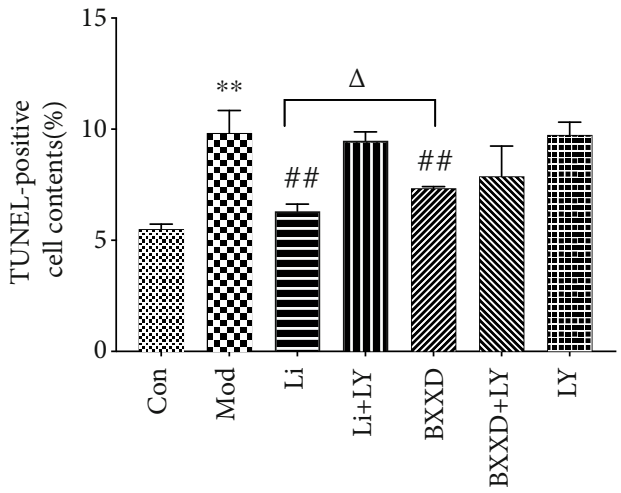

(c)

FIGURE 2: BXXD pretreatment attenuates t-BHP-induced apoptosis in MIN6 cells. Morphology of t-BHP-induced apoptosis in MIN6 cells pretreated with BXXD observed by (a) TUNEL and Hoechst staining and by (b) Hoechst staining observed under a fluorescence microscope. (c) The percentage of apoptotic cells in each group by TUNEL. MIN6 cells were incubated with DMEM (Con), DMEM plus t-BHP (Mod), DMEM, t-BHP plus liraglutide (Li), DMEM, t-BHP, liraglutide plus LY294002 (Li+LY), DMEM, t-BHP plus BXXD $0.5 \mathrm{mg} / \mathrm{ml}$ (BXXD), and DMEM, t-BHP, BXXD $0.5 \mathrm{mg} / \mathrm{ml}$ plus LY294002 (BXXD+LY). Values are expressed as mean \pm SEM. The experiment was repeated three times. ${ }^{*} P<0.05$ versus control group; ${ }^{* *} P<0.01$ versus control group; ${ }^{*} P<0.05$ versus model group; ${ }^{\#} P<0.01$ versus model group; ${ }^{\triangle} P<0.05 \mathrm{BXXD}$ versus Li group; and ${ }^{\triangle} \triangle P<0.01 \mathrm{BXXD}$ versus Li group. 




FIGURE 3: Effect of BXXD on insulin secretion. MIN6 cells were incubated with DMEM (Con), DMEM plus t-BHP (Mod), DMEM, t-BHP plus liraglutide (Li), DMEM, t-BHP, liraglutide plus LY294002 (Li+LY), DMEM, t-BHP plus BXXD 0.5 mg/ml (BXXD), and DMEM, t-BHP, BXXD $0.5 \mathrm{mg} / \mathrm{ml}$ plus LY294002 (BXXD+LY). ${ }^{*} P<0.05$ versus $2.8 \mathrm{mM}$ Glc; ${ }^{* *} P<0.01$ versus $2.8 \mathrm{mM}$ Glc.

promote insulin release in the model group compared to the $2.8 \mathrm{mM}$ group $(7.70 \pm 1.46$ at $16.7 \mathrm{mM}$ Glc versus $5.29 \pm 1.35$ at $2.8 \mathrm{mM}$ Glc, $P>0.05)$. However, BXXD and liraglutide could effectively release insulin compared to that observed in the model group (BXXD: $9.31 \pm 1.99$ at $16.7 \mathrm{mM}$ Glc versus $5.47 \pm 1.33$ at $2.8 \mathrm{mM}$ Glc; Li: $9.22 \pm 0.86$ at $16.7 \mathrm{mM}$ Glc versus $5.98 \pm 1.46$ at $2.8 \mathrm{mM}$ Glc, $P<0.05$, respectively), indicating that the GSIS impairment by t-BHP can be reversed by BXXD and liraglutide. In addition, when LY294002 was preadded, the protective effects of BXXD and Li could be neutralized and the GSIS impairment could not be rectified.

3.4. BXXD Protected MIN6 Cells from t-BHP-Induced Oxidative Stress. To determine whether BXXD affects oxidative stress-related biochemical enzymes, the levels of oxidant and antioxidant enzymes, such as MDA, SOD, and GSH-Px, were measured. t-BHP induction markedly decreased the levels of SOD and GSH-Px and increased the level of MDA (SOD: $7.23 \pm 0.64$ versus $13.42 \pm 1.80$, GSH-Px: $214.22 \pm$ 47.26 versus $428.18 \pm 39.42$, and MDA: $261 \pm 37.27$ versus $112.12 \pm 14.08, P<0.05$, respectively). The activities of SOD and GSH-Px were significantly increased in the BXXD pretreatment group relative to the model group (SOD: $12.15 \pm 1.48$ versus $7.23 \pm 0.64$, GSH-Px: $308.26 \pm 66.19$ versus $214.22 \pm 47.26, P<0.05$, respectively). In contrast, MDA production was significantly decreased compared to that in the model group $(181.19 \pm 39.29$ versus $261 \pm 37.27, P<$ $0.05)$. These results also confirmed that the antioxidant capacity of BXXD was comparable to that of liraglutide (SOD: $12.15 \pm 1.48$ versus $10.41 \pm 1.67$, MDA: $181.19 \pm$ 39.29 versus $190.32 \pm 20.34, P>0.05)$. The effect of liraglutide on improving GSH-Px expression was more potent than that of BXXD $(308.26 \pm 66.19$ versus $381.72 \pm 25.28, P<$ $0.05)$. Furthermore, BXXD and liraglutide in the presence of PI3K inhibitor LY294002 could not inhibit oxidative stress more effectively than that in the model group (Figure 4).
3.5. BXXD Attenuated Intracellular ROS Generation. The generation of intracellular ROS induced by $\mathrm{t}$-BHP promotes cellular damage. To determine whether BXXD attenuated cell apoptosis by reducing ROS generation, intracellular ROS concentrations were measured using the DCFH-DA assay. Intracellular ROS generation was significantly increased in t-BHP-treated MIN6 cells compared to nontreated MIN6 cells $(53760.75 \pm 5057.22$ versus $32003.25 \pm$ $1029, P<0.05)$. However, ROS generation was significantly reduced by BXXD and liraglutide pretreatment (BXXD: $33992.25 \pm 4655.02$ versus $53760.75 \pm 5057.22$, Li: $35003 \pm$ 5131.53 versus $53760.75 \pm 5057.22, P<0.05$, respectively). BXXD and liraglutide in the presence of PI3K inhibitor LY294002 could not decrease ROS generation more effectively than that in the model group (Figure 5).

3.6. BXXD Protected MIN6 Cells via Activation of the PI3K/AKT/FOXO1 Pathway. To identify the signaling pathway involved in the protective effects of BXXD, we tested the levels of AKT and phospho-AKT proteins in MIN6 cells using western blot. A $2 \mathrm{~h}$ incubation with $100 \mu \mathrm{M}$ t-BHP significantly decreased the phospho-AKT/AKT levels compared with that in the control group $(0.57 \pm 0.12$ versus $1.20 \pm 0.24, \quad P<0.05)$. This decrease was dramatically reversed by BXXD and liraglutide (BXXD: $1.02 \pm 0.20$ versus $0.57 \pm 0.12$, Li: $1.19 \pm 0.06$ versus $0.57 \pm 0.12, P<0.05)$, but no significant difference was found between the BXXD and liraglutide groups $(P>0.05)$. Since FOXO1 is one of the crucial downstream effectors of AKT, we next tested the levels of FOXO1 and phospho-FOXO1 proteins. Phosphorylation of FOXO1 was markedly decreased in the t-BHP group compared to the control group $(0.55 \pm 0.08$ versus $1.10 \pm 0.26$, $P<0.05)$. Besides, phospho-FOXO1/FOXO1 level was substantially attenuated in the BXXD and liraglutide groups compared to the model group (BXXD: $1.14 \pm 0.26$ versus $0.55 \pm 0.08, P<0.05$, liraglutide: $1.25 \pm 0.17$ versus $0.55 \pm$ $0.08, \quad P<0.05$, respectively); however, no discernible 


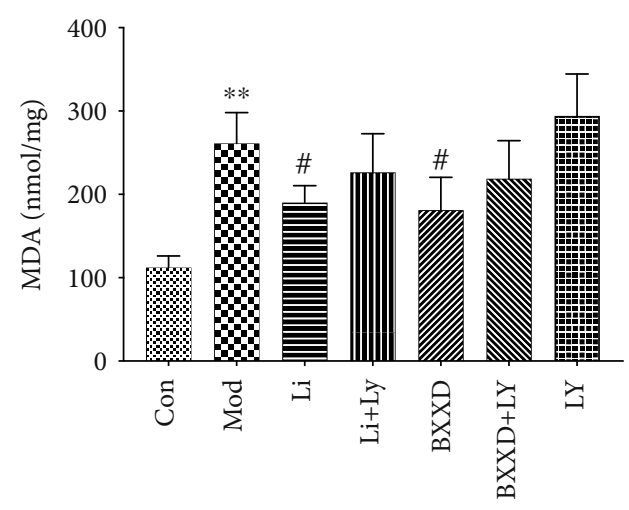

(a)

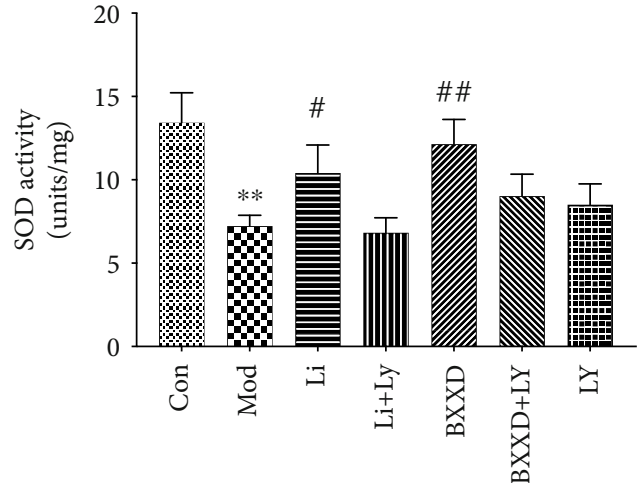

(b)

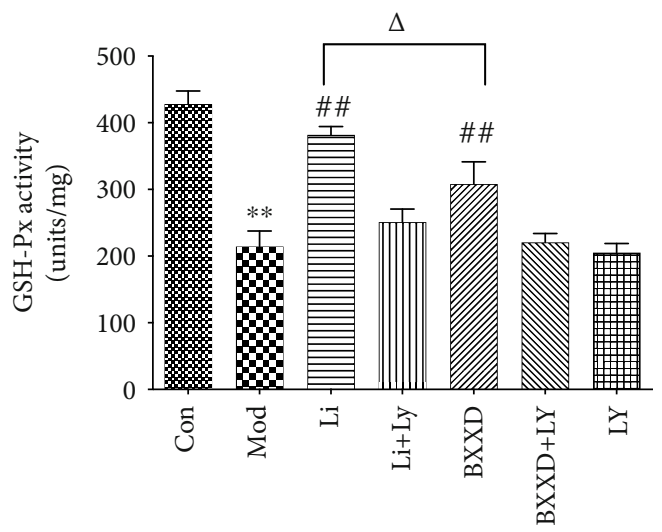

(c)

FIGURE 4: Protective effects of BXXD by regulating antioxidant function by increasing the activities of SOD and GSH-Px and decreasing the production of MDA. MIN6 cells were incubated with DMEM (Con), DMEM plus t-BHP (Mod), DMEM, t-BHP plus liraglutide (Li), DMEM, t-BHP, liraglutide plus LY294002 (Li+LY), DMEM, t-BHP plus BXXD $0.5 \mathrm{mg} / \mathrm{ml}$ (BXXD), and DMEM, t-BHP, BXXD 0.5 mg/ml plus LY294002 (BXXD+LY). Values are expressed as mean \pm SEM. The experiment was repeated three times. ${ }^{*} P<0.05$ versus control group; ${ }^{* *} P<0.01$ versus control group; ${ }^{\#} P<0.05$ versus model group; ${ }^{\# \#} P<0.01$ versus model group; ${ }^{\triangle} P<0.05 \mathrm{BXXD}$ versus $\mathrm{Li}$ group; and ${ }^{\triangle} \triangle P<0.01 \mathrm{BXXD}$ versus Li group.

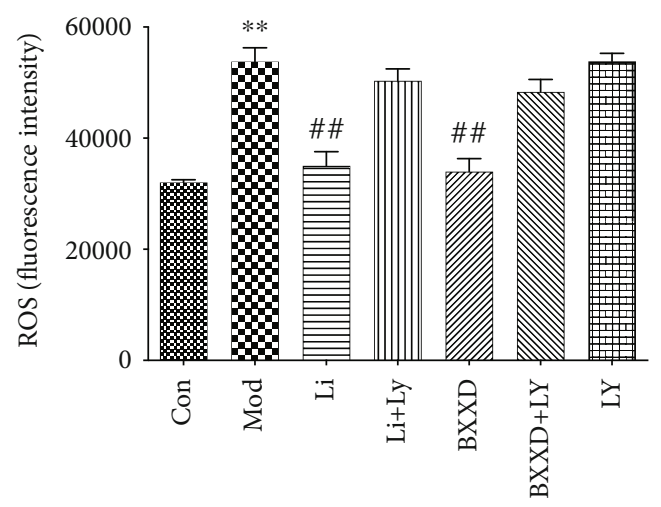

FIgURE 5: Protective effects of BXXD due to reduced t-BHP-induced generation of intracellular ROS measured by the DCFH-DA assay. MIN6 cells were incubated with DMEM (Con), DMEM plus t-BHP (Mod), DMEM, t-BHP plus liraglutide (Li), DMEM, t-BHP, liraglutide plus LY294002 (Li+LY), DMEM, t-BHP plus BXXD $0.5 \mathrm{mg} / \mathrm{ml}$ (BXXD), and DMEM, t-BHP, BXXD $0.5 \mathrm{mg} / \mathrm{ml}$ plus LY294002 (BXXD+LY). Values are expressed as mean \pm SEM. The experiment was repeated three times. ${ }^{*} P<0.05$ versus control group; ${ }^{* *} P<0.01$ versus control group; ${ }^{\#} P<0.05$ versus model group; and ${ }^{\# \#} P<0.01$ versus model group.

difference in phospho-FOXO1/FOXO1 expression was observed between the groups treated with BXXD and liraglutide $(P>0.05)$. When LY294002 was preadded, BXXD and liraglutide could not significantly increase the expres- sion of p-AKT/AKT and p-FOXO1/FOXO1. In addition, there was no significant difference between the control and LY294002-only groups, which ruled out the possible effect of LY294002 (Figure 6). 


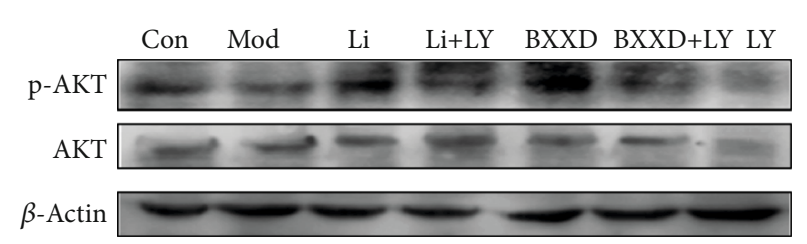

(a)

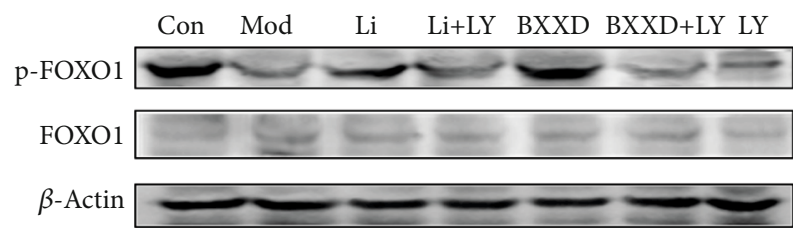

(c)

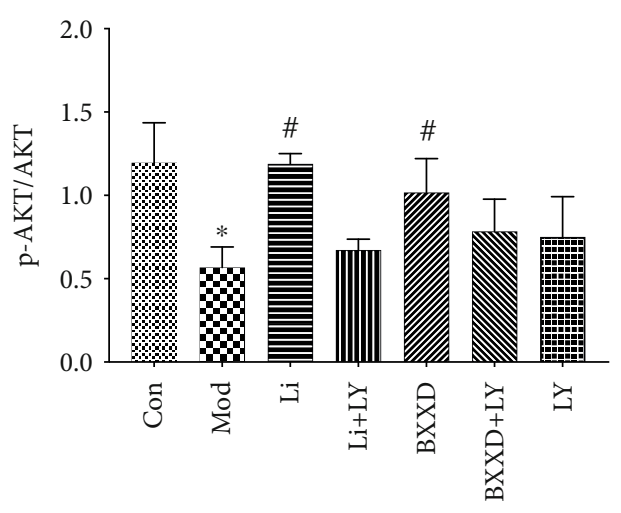

(b)

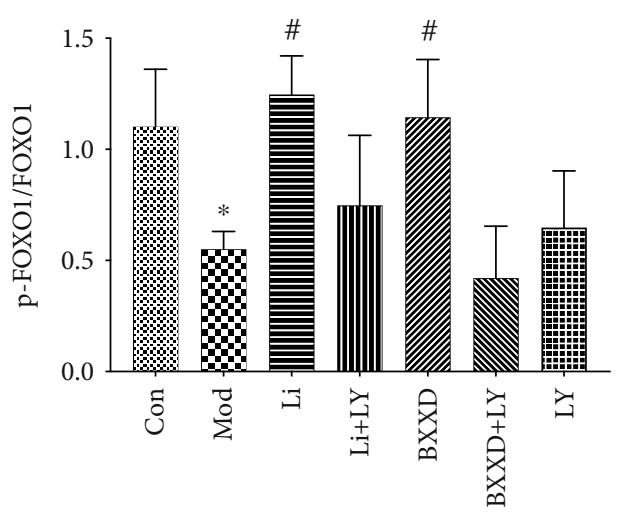

(d)

FIGURE 6: Effects of BXXD on the expression of AKT, phosphorylated AKT, FOXO1, and phosphorylated FOXO1 in MIN6 cells treated with THBP. (a, b) Western blot and quantitative measurement of AKT and phosphorylated AKT in MIN6 cells. Lane loading was normalized by reblotting with $\beta$-actin. The levels were expressed as p-AKT/AKT and normalized relative to the control group. (c, d) Western blot and quantitative measurement of FOXO1 and phosphorylated FOXO1 in MIN6 cells. Lane loading was normalized by reblotting with $\beta$-actin. The levels were expressed as p-AKT/AKT and normalized relative to the control group. MIN6 cells were incubated with DMEM (Con), DMEM plus t-BHP (Mod), DMEM, t-BHP plus liraglutide (Li), DMEM, t-BHP, liraglutide plus LY294002 (Li+LY), DMEM, t-BHP plus BXXD $0.5 \mathrm{mg} / \mathrm{ml}(\mathrm{BXXD})$, and DMEM, t-BHP, BXXD $0.5 \mathrm{mg} / \mathrm{ml}$ plus LY294002 (BXXD+LY). ${ }^{*} P<0.05$ versus control group; ${ }^{\#} P<0.05$ versus model group.

3.7. BXXD Downregulated the Expression of Bax, P27, and Caspase-3. The proapoptotic Bcl-2 family member Bax and the cell cycle inhibitor P27 have been reported to be immediate target genes of FOXO1. The best recognized biochemical hallmark of both early and late stages of apoptosis is the activation of caspases, and Caspase-3 activation is a critical determinant of apoptosis. Bax, P27, and Caspase-3 expression levels were examined in order to clarify further the molecular mechanism underlying the BXXD-mediated inhibition of apoptosis. The expression of Bax, P27, and Caspase3 was markedly upregulated in the t-BHP group compared with the control group (Bax: $1.04 \pm 0.13$ versus $0.41 \pm 0.24$, P27: $1.51 \pm 0.25$ versus $0.85 \pm 0.10$, and Caspase- $3: 1.18 \pm$ 0.31 versus $0.55 \pm 0.26, P<0.05$, respectively). Bax, P27, and Caspase-3 expression levels were markedly downregulated in the BXXD group compared with the t-BHP group (Bax: $0.42 \pm 0.34$ versus $1.04 \pm 0.13$, P27: $0.79 \pm 0.26$ versus $1.51 \pm 0.25$, and Caspase- 3 : $0.63 \pm 0.19$ versus $1.18 \pm 0.31$, $P<0.05$, respectively). A noticeable decrease in the expression of Bax, P27, and Caspase-3 was also observed in the liraglutide group compared to the t-BHP group (Bax: $0.50 \pm 0.40$ versus $1.04 \pm 0.13, \mathrm{P} 27: 0.93 \pm 0.06$ versus
$1.51 \pm 0.25$, and Caspase-3: $0.62 \pm 0.25$ versus $1.18 \pm 0.31$, $P<0.05$, respectively). No significant difference was found between the BXXD and liraglutide groups $(P>0.05)$. When the PI3K inhibitor LY294002 was preadded, liraglutide could not decrease the expression of Bax, Caspase-3, and P27, and BXXD could not decrease the expression of Bax and Caspase-3 (Figure 7).

\section{Discussion}

BXXD reportedly promotes insulin sensitivity, improves insulin resistance of peripheral target tissues (liver, adipose tissue, and skeletal muscle), and regulates glycolipid metabolism [19-24]. However, the molecular mechanism underlying BXXD effect on $\beta$-cells is not fully understood. Here, we found that BXXD protected MIN6 pancreatic $\beta$-cells by inhibiting t-BHP-induced apoptosis and promoting glucose-induced insulin secretion. The mechanisms mediating these effects may be related to the restoration of the PI3K/AKT signaling pathway and its downstream target forkhead box O1 (FOXO1). Additionally, BXXD significantly attenuated oxidative stress and inhibited the 


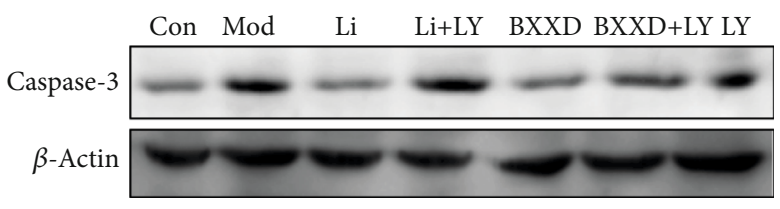

(a)

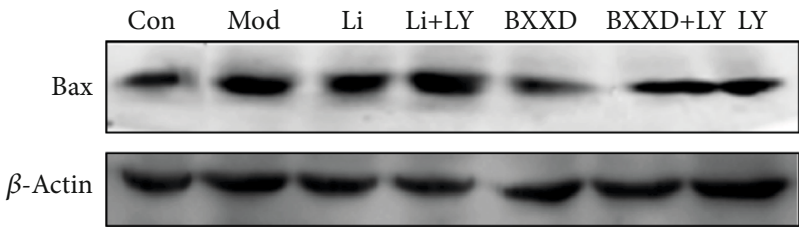

(c)

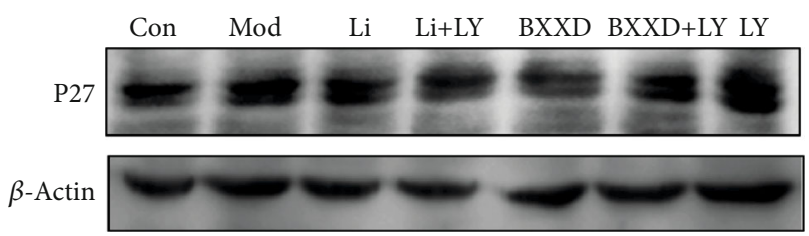

(e)



(b)



(d)

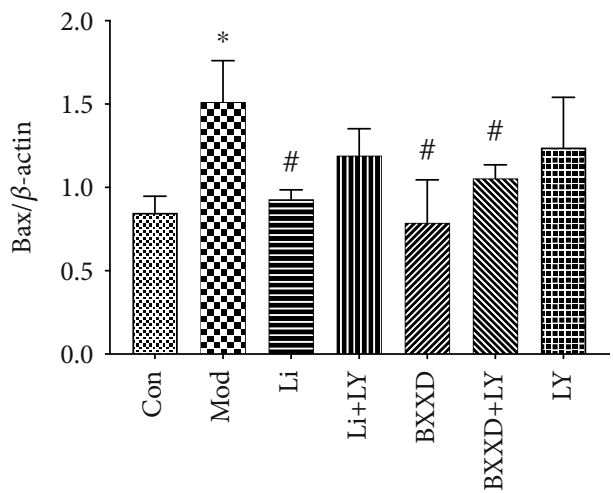

(f)

Figure 7: Effects of BXXD on the expression of Bax, P27, and Caspase-3 in MIN6 cells treated with THBP. (a, b) Western blot and quantitative measurement of Caspase-3. (c, d) Western blot and quantitative measurement of Bax. (e, f) Western blot and quantitative measurement of P27. Lane loading was normalized by reblotting with $\beta$-actin. MIN6 cells were incubated with DMEM (Con), DMEM plus t-BHP (Mod), DMEM, t-BHP plus liraglutide (Li), DMEM, t-BHP, liraglutide plus LY294002 (Li+LY), DMEM, t-BHP plus BXXD $0.5 \mathrm{mg} / \mathrm{ml}$ (BXXD), and DMEM, t-BHP, BXXD $0.5 \mathrm{mg} / \mathrm{ml}$ plus LY294002 (BXXD+LY). Data was expressed as mean \pm SD and shown as a percentage of the control group. ${ }^{*} P<0.05$ versus control group; ${ }^{\#} P<0.05$ versus model group.

expression of related apoptotic indicators Bcl-2-associated X protein (Bax), protein 27 (P27), and cysteine aspartic acidspecific protease 3 (Caspase-3) (Figure 7).

Generally, T2DM is characterized by hyperglycemia and elevated free fatty acid levels, which contribute to the dysfunction and death of pancreatic $\beta$-cells and lead to insufficient insulin secretion $[25,26]$. Oxidative stress damage and multiple-induced caspase cascade may be two crucial factors resulting in pancreatic $\beta$-cell apoptosis. Persistent hyperglycemia induces ROS formation, leading to increased lipid peroxidation level and/or decreased antioxidase activity, thereby triggering oxidative stress [27, 28]. High levels of ROS and reactive nitrogen produced during oxidative stress can lead to cell dysfunction and death through lipid peroxidation, DNA damage, and cell apoptosis regulation [29]. tBHP is a short-chain analog of lipid hydroperoxides, which mimics the toxic effect of peroxidized fatty acids. t-BHP has been reported to induce apoptosis in vivo and in vitro [29]. In agreement with previous studies, our results showed that $100 \mu \mathrm{M} \mathrm{t}$-BHP notably induced apoptosis [30]. Here, we 
provide evidence that BXXD treatment could inhibit t-BHPinduced apoptosis.

Oxidative stress is marked by the imbalance between the oxidative system and the antioxidant system, toward the oxidative system, which causes damage to the organism [31]. $\beta$-cell apoptosis lies at the core of DM pathophysiology, and oxidative stress is a mediator of $\beta$-cell apoptosis [32]. ROS and MDA usually represent oxidative stress indicators, while SOD and GSH-Px are common antioxidant enzymes. Excessive ROS production is closely related to the apoptosis of pancreatic $\beta$-cells and is the fundamental cause of insulin secretion and utilization disorders [33]. Antioxidant enzymes, such as SOD and GSH-Px, participate in ROS homeostasis. SOD is the first antioxidant enzyme against ROS; it converts $\mathrm{O}_{2}^{-}$into $\mathrm{H}_{2} \mathrm{O}_{2}$, which is further degraded into $\mathrm{O}_{2}$ and $\mathrm{H}_{2} \mathrm{O}$ by GSH-Px. GSHPx catalyzes the hydrogen peroxide reduction by two molecules of glutathione, a part of the ROS defense system. MDA is generated in vivo via the peroxidation of polyunsaturated fatty acids. MDA interacts with proteins and is itself potentially atherogenic. Our study showed an increase in ROS formation and lipid peroxidation in terms of MDA levels and a decrease in antioxidase activity in terms of SOD and GSH-Px in t-BHP-induced MIN6 cells. BXXD significantly attenuated intracellular ROS generation, decreased MDA levels, and increased SOD and GSH-Px levels, which might be one of the mechanisms underlying the attenuation of oxidative stress in MIN6 cells.

The PI3K/AKT signaling pathway plays a pivotal role in regulating cell growth, proliferation, survival, and metabolism. Phosphatidylinositol 3-kinase (PI3K) can be activated by numerous $\beta$-cell surface receptors, and Protein Kinase B (AKT) is one of the pivotal PI3K effectors [34]. FOXO1 is a key factor located downstream of the PI3K/AKT pathway, and its transcriptional activity is regulated by phosphorylation of AKT [35]. AKT activation is accompanied by phosphorylation of proapoptotic Bad protein and nuclear FOXOs, hence suppression of FOXO1/3a transcription factors, which are known to be important for pancreatic $\beta$-cell survival [36]. This study investigated the effects of BXXD on t-BHP-induced PI3K/AKT insulin signaling in MIN6 cells and whether BXXD can improve the insulin secretion function of islet $\beta$-cells through this pathway. The results showed that t-BHP inhibited the serine phosphorylation of AKT and FOXO1 in MIN6 cells. Cotreatment with BXXD reversed the inhibitory effect of $t-B H P$ on AKT and FOXO1 phosphorylation. Furthermore, coadministration of PI3K inhibitor LY294002 with BXXD abolished the BXXD-induced increase in AKT and FOXO1 phosphorylation levels. We also found that BXXD can improve the insulin secretion of islet $\beta$-cells stimulated by glucose; this effect could also be reversed after the addition of LY294002. This finding suggests that BXXD improves the secretory function of islet $\beta$-cells by promoting the transduction of the PI3K/AKT/FOXO1 insulin signaling pathway.

FOXO transcription factors can regulate numerous target genes such as cell cycle inhibitor $\mathrm{p} 27$, the Bcl-2 protein family $[37,38]$. In order to further explore the PI3K/AKT/FOXO1 signaling pathway-related downstream factors and the pro-

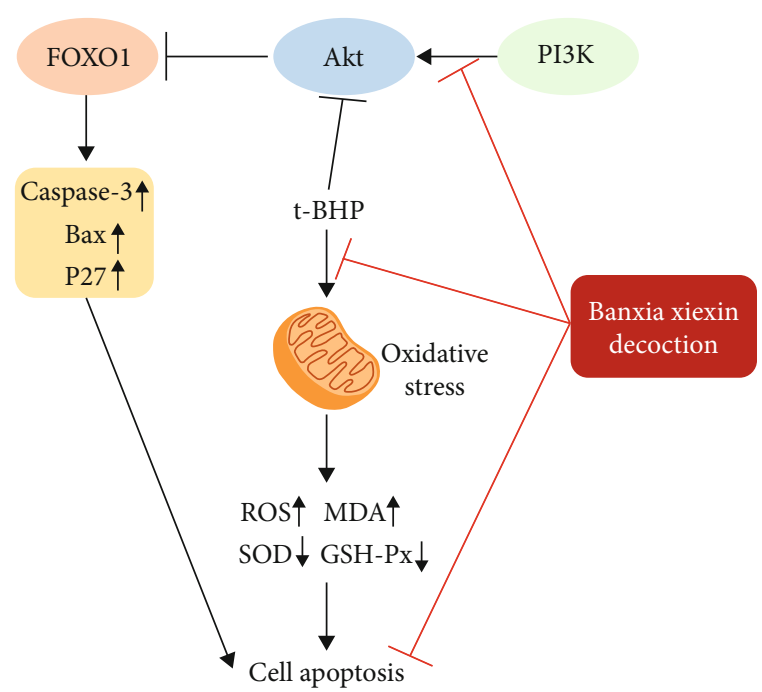

FIgURE 8: Schematic illustration of possible mechanisms of BXXD inhibited t-BHP-induced apoptosis. Protective function of BXXD for MIN6 cells against t-BHP-induced oxidative damage and apoptosis and improved insulin secretory was activation of the $\mathrm{PI} 3 \mathrm{~K} / \mathrm{AKT} / \mathrm{FOXO1}$ pathway.

tective effect of BXXD on MIN6, we screened several apoptosis-related proteins. The $\mathrm{Bcl}-2$ protein family plays a key role in controlling the mitochondrial pathway of $\beta$-cell apoptosis and the activation of caspases [29]. Bcl-2-related proteins fall into two groups that generally either promote apoptosis (Bax, Bak, and Bad) or repress apoptosis (Bcl-2 and $\mathrm{Bcl}-\mathrm{xL}$ ). Bax is a critical regulator of the mitochondrial apoptotic pathway. Increased Bax expression, which upregulates Caspase-3, was recently reported to correlate with $\beta$-cell apoptosis. P27, a member of the cyclin-dependent kinase inhibitor (CDKI) family, is highly expressed in $\beta$-cell nuclei and plays essential roles during G1-to-S phase progression. Moreover, p27-deleted mice displayed improved glucose tolerance and increased insulin secretion, which was attributed to the increased islet mass [39]. In the current study, when apoptosis occurred, the gene expression of Bax, P27, and Caspase- 3 increased significantly. Furthermore, when BXXD attenuated the apoptosis, the expression of Bax, P27, and Caspase- 3 decreased, indicating that these related apoptotic indicators may be involved in the process by which BXXD protects cells against ROS-induced oxidative damage.

This study has some limitations. The molecular targets of BXXD may not be only dependent on the PI3K/AKT/FOXO1 signaling pathway; the possibility of other mechanisms cannot be excluded. Further studies are required to explore the mechanism of action of BXXD in detail.

\section{Conclusions}

BXXD protected MIN6 cells against t-BHP-induced oxidative damage and apoptosis and improved insulin secretory function through modulation of the PI3K/AKT/FOXO1 pathway (Figure 8). Thus, BXXD might be developed as a therapeutic agent for T2DM. 


\section{Abbreviations}

$\begin{array}{ll}\text { DM: } & \text { Diabetes mellitus } \\ \text { T2DM: } & \text { Type 2 diabetes mellitus } \\ \text { t-BHP: } & \text { tert-Butyl hydroperoxide } \\ \text { Li: } & \text { Liraglutide } \\ \text { LY: } & \text { PI3K inhibitor LY294002 } \\ \text { BXXD: } & \text { Banxia Xiexin Decoction } \\ \text { ATCC: } & \text { American Type Culture Collection } \\ \text { DMSO: } & \text { Dimethylsulfoxide } \\ \text { DMEM: } & \text { Dulbecco's modified Eagle's medium } \\ \text { FBS: } & \text { Fetal bovine serum } \\ \text { PBS: } & \text { Phosphate-buffered saline } \\ \text { MTT: } & \text { 3-(4,5-Dimethylthiazol-2-yl)-2,5-diphenyltet- } \\ & \text { razolium bromide } \\ \text { TUNEL: } & \text { Terminal deoxynucleotidyl transferase dUTP } \\ & \text { nick end labeling assay } \\ \text { DAPI: } & \text { 4'6-Diamidino-2-phenylindole } \\ \text { KRB: } & \text { Krebs-Ringer bicarbonate } \\ \text { MDA: } & \text { Malondialdehyde } \\ \text { SOD: } & \text { Superoxide dismutase } \\ \text { GSH-Px: } & \text { Glutathione peroxidase } \\ \text { ROS: } & \text { Reactive oxygen species } \\ \text { DCFH-DA: } & \text { Dichloro-dihydro-fluorescein diacetate } \\ \text { PBS: } & \text { Phosphate-buffered saline } \\ \text { RIPA: } & \text { Radioimmunoprecipitation assay } \\ \text { SDS-PAGE: } & \text { Sodium dodecyl sulfate-polyacrylamide gel } \\ & \text { electrophoresis } \\ \text { PVDF: } & \text { Polyvinylidene fluoride } \\ \text { TBS: } & \text { Tris-buffered saline } \\ \text { PI3K: } & \text { Phosphatidylinositol 3-kinase } \\ \text { AKT: } & \text { Protein Kinase B } \\ \text { FOXO1: } & \text { Forkhead box O1 } \\ \text { Caspase-3: } & \text { Cysteine aspartic acid-specific protease 3 } \\ \text { Bcl-2: } & \text { B cell lymphoma/leukemia-2 } \\ \text { Bax: } & \text { Bcl-2-associated X protein } \\ \text { p27: } & \text { Protein 27 } \\ \text { BCA: } & \text { Bicinchoninic acid } \\ \text { ANOVA: } & \text { One-way analysis of variance. } \\ & \end{array}$

\section{Data Availability}

The data sets used or analyzed during the study are available from the corresponding author on reasonable request.

\section{Conflicts of Interest}

No competing financial interests existed.

\section{Authors' Contributions}

NQ, SM, DLJ, and PB conceived the study and participated in its design. DLJ and PB participated in the cell viability and apoptosis analysis, western blot study. TYM, YYN, PQ, and ZMZ performed the statistical analysis. DLJ and PB wrote the initial manuscript; NQ and SM helped to revise it critically. DLJ and PB are co-first authors. Min Sun and Qing $\mathrm{Ni}$ are co-corresponding authors. All authors read and approved the final manuscript. Li-juan Du and Bing Pang contributed equally to this work.

\section{Acknowledgments}

This study is supported by grants from the National Natural Science Foundation of China (81373594), from the special program for excellent scientific personnel training of China Academy of Traditional Chinese Medicine (ZZ13-YQ-032), and from the Institutional Research Foundation of Guang'anmen Hospital, China Academy of Chinese Medical Sciences (59957).

\section{References}

[1] S. Bajaj, "RSSDI clinical practice recommendations for the management of type 2 diabetes mellitus 2017," International Journal of Diabetes in Developing Countries, vol. 38, Suppl 1, pp. 1-115, 2018.

[2] K. Ogurtsova, J. D. da Rocha Fernandes, Y. Huang et al., "IDF Diabetes Atlas: global estimates for the prevalence of diabetes for 2015 and 2040," Diabetes Research and Clinical Practice, vol. 128, no. 6, pp. 40-50, 2017.

[3] Association $\mathrm{AD}$ 10, "Microvascular complications and foot care: standards of medical care in diabetes-2018," Diabetes Care, vol. 41, (Suppl 1):S105, 2018.

[4] L. Zhang, J. Long, W. Jiang et al., "Trends in chronic kidney disease in China," The New England Journal of Medicine, vol. 375, no. 9, pp. 905-906, 2016.

[5] Y. Jiang, X. Ran, L. Jia et al., "Epidemiology of type 2 diabetic foot problems and predictive factors for amputation in China," The International Journal of Lower Extremity Wounds, vol. 14, no. 1, pp. 19-27, 2015.

[6] J. M. Martinez-Moreno and M. Garciacaballero, "Influences of the diabetes surgery on pancreatic beta-cells mass," Nutricion Hospitalaria, vol. 28, Suppl 2, pp. 88-94, 2013.

[7] S. Costes, R. Langen, T. Gurlo, A. V. Matveyenko, and P. C. Butler, "Cell failure in type 2 diabetes: a case of asking too much of too few?," Diabetes Care, vol. 62, no. 2, pp. 327-335, 2013.

[8] Y. Zhang, F. Yin, J. Liu, and Y. Wang, "Geniposide protects pancreatic INS-1E $\beta$ cells from hIAPP-induced cell damage: potential involvement of insulin degrading-enzyme," Cell Biology International, vol. 39, no. 4, pp. 373-378, 2015.

[9] D. H. Kim, M. H. Park, E. K. Lee et al., "The roles of FoxOs in modulation of aging by calorie restriction," Biogerontology, vol. 16, no. 1, pp. 1-14, 2015.

[10] P. Xia and X. Y. Xu, "PI3k/AKT/mTOR signaling pathway in cancer stem cells: from basic research to clinical application," American Journal of Cancer Research, vol. 5, no. 5, pp. 16021609, 2015.

[11] Z. Nozhat and M. Hedayati, "PI3K/AKT pathway and its mediators in thyroid carcinomas," Molecular Diagnosis \& Therapy, vol. 20, no. 1, pp. 13-26, 2016.

[12] J. Bertacchini, N. Heidari, L. Mediani et al., "Targeting PI3K/AKT/mTOR network for treatment of leukemia," Cellular and Molecular Life Sciences, vol. 72, no. 12, pp. 2337-2347, 2015.

[13] W. Na, "Clinical observation on treating $\mathrm{Hp}$ infection in chronic gastritis with the Banxia Xiexin decoction plus western 
medicine," Clinical Journal of Chinese Medicine, vol. 11, no. 17, pp. 30-32, 2019.

[14] L. I. Jing-jie and W. Fang, "Observation on curative effect of BanXia XieXin decoction on 20 cases of upper gastrointestinal trac reaction after cisplatin chemotherapy for gastric cancer," Xinjiang Journal of Traditional Chinese Medicine, vol. 37, no. 4, pp. 13-15, 2019.

[15] H. A. N. Songlin, L. I. Shiyun, and L. Junfeng, "Clinical observation on Banxia Xiexin decoction in the treatment of diabetes complicated with gastroparesis," Guangming Journal of Chinese Medicine, vol. 33, no. 1, pp. 59-61, 2018.

[16] X. Wang, Y. Guangjun, and L. Chong, "Effects of Banxia Xiexin decoction on chronic atrophic gastritis of spleenstomach damp-heat type and serum inflammatory index and immune function," World Chinese Medicine, vol. 14, no. 2, pp. 412-416, 2019.

[17] N. Qing, M. X. Du Lijuan, and T. Minghong, "Preliminary exploration of effect for Banxia Xiexin decoction on plasma GLP-1 in newly diagnosed type 2 diabetes patients," Beijing Journal of Traditional Chinese Medicine, vol. 36, no. 6, pp. 549-551, 2017.

[18] N. I. Qing, M. Xiang, and D. Li-juan, "Effects of Banxia Xiexin decoction on $\mathrm{H}_{2} \mathrm{O}_{2}$-induced RINm-5F cell apoptosis and insulin secretion," Beijing Journal of Traditional Chinese Medicine, vol. 36, no. 12, 2017.

[19] LW S, LJ D, Q N, Theoretical discussion and clinical application of Banxia Xiexin decoction in the treatment of diabetes, vol. 59, no. 3, 2018Zhong Yi Za Zhi, 2018.

[20] Z. Ss, A. Zz, and Y. Bh, "The effects of modified Banxia Xiexin decoction on blood glucose, blood lipid and islet cell function in rats with type 2 diabetes mellitus," Journal of Hubei University of Chinese Medicine, vol. 19, no. 5, pp. 13-17, 2017.

[21] SS Z, Effect of modified Banxia Xiexin decoction on pancreatic PERK-CHOP apoptosis pathway in T2DM rats, Guangzhou University of Chinese Medicine, 2017, (Dissertation)..

[22] D. Ht, "Clinical application of Banxia Xiexin decoction in the treatment of diabetes mellitus," World Latest Medicine Information (Electronic Version), vol. 18, no. 77, pp. 184-195, 2018.

[23] JY Y, Effect of Banxia Xiexin decoction on insulin resistance and expression of IL-6, TNF- $\alpha, F F A$ and NO in type 2 diabetic rats, Chengdu University of Chinese Medicine, 2017, (Dissertation).

[24] S L, "Clinical observation of combination of Banxia Xiexin decoction and western medicine in the treatment of T2DM," China's Naturopathy, vol. 27, no. 13, pp. 51-52, 2019.

[25] A. T. Templin, T. Samarasekera, D. T. Meier et al., "Apoptosis repressor with caspase recruitment domain ameliorates amyloid-induced $\beta$-cell apoptosis and JNK pathway activation," Diabetes, vol. 66, no. 10, pp. 2636-2645, 2017.

[26] H.-t. Xiao, B. Wen, Z.-w. Ning et al., "Cyclocarya paliurus tea leaves enhances pancreatic $\beta$ cell preservation through inhibition of apoptosis," Scientific Reports, vol. 7, no. 1, p. 9155, 2017.

[27] C. Holze, C. Michaudel, C. Mackowiak et al., "Oxeiptosis, a ROS-induced caspase-independent apoptosis-like cell-death pathway," Nature Immunology, vol. 19, no. 2, pp. 130-140, 2018.

[28] T. Fiorentino, A. Prioletta, P. Zuo, and F. Folli, "Hyperglycemia-induced oxidative stress and its role in diabetes mellitus related cardiovascular diseases," Current Pharmaceutical Design, vol. 19, no. 32, pp. 5695-5703, 2013.
[29] Y.-G. Zhu, X.-C. Chen, Z.-Z. Chen et al., "Curcumin protects mitochondria from oxidative damage and attenuates apoptosis in cortical neurons," Acta Pharmacologica Sinica, vol. 25, no. 12, pp. 1606-1612, 2004.

[30] E. Chang, "1, 25-Dihydroxyvitamin D decreases tertiary butylhydrogen peroxide-induced oxidative stress and increases AMPK/SIRT1 activation in C2C12 muscle cells," Molecules, vol. 24, no. 21, p. 3903, 2019.

[31] K. Kannan and S. K. Jain, "Oxidative stress and apoptosis," Pathophysiology, vol. 7, no. 3, pp. 153-163, 2000.

[32] R. P. Robertson and J. S. Harmon, "Diabetes, glucose toxicity, and oxidative stress: A case of double jeopardy for the pancreatic islet $\beta$ cell," Free Radical Biology and Medicine, vol. 41, no. 2, pp. 177-184, 2006.

[33] G. Chen, X. Yang, X. Yang et al., "Jia-Wei-Jiao-Tai-Wan ameliorates type 2 diabetes by improving $\beta$ cell function and reducing insulin resistance in diabetic rats," BMC Complementary and Alternative Medicine, vol. 17, no. 1, p. 507, 2017.

[34] M. Szydłowski, E. Jabłońska, and P. Juszczyński, "FOXO1 transcription factor: a critical effector of the PI3K-AKT axis in B-cell development," International Reviews of Immunology, vol. 33, no. 2, pp. 146-157, 2014.

[35] A. Brunet, A. Bonni, M. J. Zigmond et al., "Akt promotes cell survival by phosphorylating and inhibiting a Forkhead transcription factor," Cell, vol. 96, no. 6, pp. 857-868, 1999.

[36] L. Elghazi and E. Bernal-Mizrachi, "Akt and PTEN: $\beta$-cell mass and pancreas plasticity," Trends in Endocrinology \& Metabolism, vol. 20, no. 5, pp. 243-251, 2009.

[37] P. F. Dijkers, R. H. Medema†, J.-W. J. Lammers, L. Koenderman, and P. J. Coffer, "Expression of the proapoptotic Bcl-2 family member Bim is regulated by the forkhead transcription factor FKHR-L1," Current Biology, vol. 10, no. 19, pp. 1201-1204, 2000.

[38] P. F. Dijkers, R. H. Medema, C. Pals et al., "Forkhead transcription factor FKHR-L1 modulates cytokine-dependent transcriptional regulation of p27(KIP1)," Molecular and Cellular Biology, vol. 20, no. 24, pp. 9138-9148, 2000.

[39] S. Shao, M. Nie, C. Chen et al., "Protective action of liraglutide in beta cells under lipotoxic stress via PI3K/Akt/FoxO1 pathway," Journal of Cellular Biochemistry, vol. 115, no. 6, pp. 1166-1175, 2014. 